\title{
Diet quality and its relationship with body weight status and laboratory parameters in breast cancer patients undergoing treatment
}

Krystal Ng LS

Universiti Putra Malaysia

Chan Yoke Mun ( $\nabla$ yokemun_chan@yahoo.com )

Universiti Putra Malaysia

Zalilah MS

Universiti Putra Malaysia

\section{Research article}

Keywords: Breast cancer, Diet quality, Overweight, Serum haemoglobin

Posted Date: August 3rd, 2019

DOI: https://doi.org/10.21203/rs.2.11380/v1

License: () (i) This work is licensed under a Creative Commons Attribution 4.0 International License. Read Full License 


\section{Abstract}

Background To determine whether diet quality was associated with body weight status and laboratory parameters among breast cancer patients undergoing treatment. Methods A cross sectional study comprises of 169 patients was conducted from January to May 2017. Diet quality and level of physical activity of patients were ascertained using Healthy Eating Index (HEI)-2015 and International Physical Activity Questionnaire (IPAQ)-short form, respectively. Data were available on body mass index and biochemical data, including of serum albumin, serum hemoblogbin and neutrophils count. Logistic regression was performed to identify the association between diet quality with body weight status, serum albumin, serum hemoglobin and neutrophils count. Results The mean total HEl score (standard deviation) of the breast cancer patients was 63.86 (8.75). More than $80 \%$ of the subjects did not adhere to the dietary guidelines for whole grains, dairy, refined grains, ratio of poly- and mono-unsaturated fatty acids to saturated fatty acids components. In the multivariate model, those who perceived higher score of total HEl-2015 were less likely to experience overweight (Q4 vs Q1: OR=0.36; 95\% Cl=0.13, 0.99) while reported higher concentration of serum hemoglobin (Q4 vs Q1: OR=0.36; $95 \% \mathrm{Cl}=0.13,0.98$; Q3 vs Q1: OR=0.35; $95 \% \mathrm{Cl}=0.13,0.92$ ). Non-significant associations of diet quality were shown for serum albumin and neutrophils count. Conclusions A majority of breast cancer patients require dietary modification, especially for fatty acids, grains and dairy products. A better diet quality was related with lower likelihood of overweight and low level of hemoglobin. Improving the quality of cancer care in breast cancer patients should emphasize on dietary intervention.

\section{Background}

Cancer- and treatment-related factors have resulted in various undesirable health outcomes among the cancer patients. Unlike other cancers such as head and neck, gastrointestinal tract, liver and lung cancers, patients with breast cancer have lower risk of undernutrition ${ }^{1,2}$. Weight gain is the common issue among breast cancer patients undergoing treatment ${ }^{3}$, which may have detrimental effect on survival as a result of inflammation ${ }^{4,5}$. In addition, breast cancer patients often suffer from anemia and neutropenia ${ }^{6}$, leads to poor prognosis. While low serum hemoglobin is related to increased level of fatigue, reduced ability to perform daily tasks and impaired quality of life ${ }^{6,7}$, an abnormally low neutrophils count predisposes cancer patients to bacterial infection, which could be lifethreatening ${ }^{8}$. Such negative impact of altered blood readings on patients' prognosis might indirectly influence treatment compliance, in light of close linkage between quality of life and treatment compliance ${ }^{9}$.

Diet is an established modifiable factor of body weight via principle of energy imbalance. Assessment of diet quality provides a better understanding on the overall picture of diet consumed in accordance with dietary guidelines, rather than depend on single nutrients ${ }^{10}$. This tool has been extensively used for epidemiological studies, in regard to the risk prediction of various health outcomes ${ }^{10}$. In addition to the reduction in cancer risk ${ }^{11}$, healthier diet was found to associate with reduced inflammation ${ }^{12}$ and improved prognosis among breast cancer survivors ${ }^{13,14}$. The health benefit of diet could be explained by the food synergy concept ${ }^{15}$, in which each of the nutrients acts simultaneously to yield an effective biological pathway. Conversely, Wu (2016) highlighted that insufficient protein intake could solely compromise the immune function ${ }^{16}$, which may forsake such notion that an evaluation of combined food is more informative in regards to a particular health issue. Nevertheless, how diet quality may associate with overweight and altered biochemical parameters for breast cancer patients, at the critical phase of on-going treatment is relatively sparse.

As poor diet is commonly perceived to be linked with cancer developement ${ }^{17,18}$, cancer patients are likely to consume low fat, high fiber diet upon diagnosis $^{19,20}$. Nonetheless, few studies highlighted the reduced diet quality during treatment among breast cancer patients, which resulted from the poor adherence to dietary guidelines for the components of fruits and vegetables ${ }^{21,22}$, grains, solid fats, alcohol and added sugars ${ }^{21}$. This could be explained by the altered taste or increased eating pleasure for "heavy taste" food during treatment ${ }^{23}$. Preference for spicy and salty food was found to associate with overweight in breast cancer patients, in light of its potential impact on increased overall appetite via oral sensory stimulation ${ }^{23}$. Diet quality covers the evaluation of a combination of food groups and nutrients from the whole food consumed, whereby the diet-disease relationship could be more detectable ${ }^{10}$. This is very beneficial for the successful implementation of dietary strategies.

A balanced diet could be an impetus for alleviated side effects which resulting from cancer or its treatment. To date, there are limited studies on diet quality in relation to weight status and abnormal laboratory variables particularly for serum albumin, serum haemoglobin and neutrophils count in breast cancer patients receiving treatment. This study sought to determine the diet quality of breast cancer patients as well as its association with body weight status and treatment-related side effects of hypoalbuminemia, low serum hemoglobin and low neutrophils count.

\section{Methods \\ Design}

This was a cross sectional study.

\section{Samples}

This study was conducted among breast cancer patients in National Cancer Institute, which is located in Federal Territory of Putrajaya. A total of 179 patients with breast cancer was enrolled using purposive sampling. The inclusion criteria were female, aged $\geq 18$ years, receiving treatment ( $\geq 1 \mathrm{month}$ ) and able to provide written consent. Patients were excluded if under hospice care or have life expectancy of $\leq 6$ months, pregnant or lactating, diagnosed with severe mental disorders or reduced ability to understand or communicate with others. 


\section{Data collection}

The data collection took five months (January - May 2017) and was carried out on patients' appointment day of treatment administration. Ethic approval of this study protocol was obtained from the Ministry of Health's Research and Ethics Committee (MREC) and Ethic Committee for Research Involving Human Subject (JKEUPM), Universiti Putra Malaysia, with written informed consents obtained from all patients prior to study enrolment.

\section{Measurements}

Measurements of weight and height were taken to the nearest $0.1 \mathrm{~kg}$ and $0.1 \mathrm{~cm}$, respectively, by using Detecto bariatric scale (6857DHR). Weight status of patients was categorized as underweight, normal, pre-obese and obesity, as represented by body mass index (BMI) below $18.50 \mathrm{~kg} / \mathrm{m}^{2}, 18.50-24.99 \mathrm{~kg} / \mathrm{m}^{2}$, $25.00-29.99 \mathrm{~kg} / \mathrm{m}^{2}$ and $\geq 30 \mathrm{~kg} / \mathrm{m}^{2}$, respectively ${ }^{24}$.

Habitual dietary intake of patients over the past one month was assessed using a 165-items semi-quantitative Malaysians' Food Frequency Questionnaires $\left(\right.$ FFQ) ${ }^{25}$. Dietary data were analysed using Nutritionist Pro version 4.0.0 (Axxya system, 2017), whereby the main database used was Malaysian food composition, and supplemented by Singaporean and USDA database. Food consumption data was transformed into Healthy eating index-2015 (HEI-2015) scoring according to nine adequacy and four moderation components ${ }^{26}$. Each food component was scored proportionately between the minimum and maximum standard as shown in Table 1. The summation of individual components yields a total HEl-2015 score, with higher score indicates better adherence to Dietary Guidelines for Americans $2015-2020^{26}$. The total HEl-2015 score was divided into quartiles. In view of the lack of well-validated diet quality tool for Malaysians ${ }^{27}$, the decision of utilizing HEl-2015 tool was made to assess patients' diet quality as it covers different food groups or subgroups which enables the result to be more discussable.

International Physical Activity Questionnaire (IPAQ)-short form was used to assess level of physical activity level of patients. Total intensity level of physical activity (MET-min/week) was identified by taking consideration into frequency and duration of each activity type at one time. The higher reading of METs score indicates more intense physical activity. Patients' physical activity level was categorized into low and moderate or high according to the total frequency, duration and intensity level of physical activities that have been carried out over the past one week ${ }^{28}$.

Demographic information on age, marital status, educational level, employment status and monthly income were collected via interviewer-administered questionnaire. Patients' medical characteristics, including time since diagnosis, cancer stage, treatment modalities and comorbidities were retrieved from medical records while serum albumin, serum hemoglobin and neutrophils count were retrieved as secondary data.

\section{Statistical analysis}

A total of 16 cases were excluded from the analysis due to incomplete data or extreme outliers. All statistical analyses were performed using Statistical Software Package (IBM SPSS statistics version 22.0), with the statistical significance set at $\mathrm{p}<0.05$ (two-sided). Patients' characteristics were described as means \pm standard deviations for continuous variables or counts with percentages for categorical variables. The differences in total mean $\mathrm{HEI}-2015 \mathrm{score}$ were compared according to patients' characteristics, using analysis of variance (ANOVA) for age and month since diagnosis or chi-square test for categorical variables. Associations of $\mathrm{HEl}-2015$ score with weight status, levels of albumin, hemoglobin and neutrophils count were analyzed using binary logistic regression, controlling for demographic and medical characteristics (Model 1). Additional adjustments of moderate-to-vigorous physical activity and/or BMI were performed in Model 2, to examine any changes of observed association in the first model.

\section{Results}

Table 2 illustrated the patients' characteristics by quartiles of total HEI-2015 scores. The HEI-2015 score was poorer with longer duration of diagnosis as well as the presence of comorbidity. The overall mean (SD) of HEl score was 63.86 (8.75). In term of HEl components (Figure 1), the median scores for adequacy components of Total Fruit, Whole Fruit, Total Vegetables, Greens and Beans, Total Protein Food, Seafood and Plant Proteins, as well as moderation components of Sodium, Added Sugars and Saturated Fats achieved the maximum scores of $100 \%$. More than half of the subjects met the recommendation for above components. On the other hand, Whole Grains, Dairy, Fatty Acids and Refined Grains scored less than $25 \%$ of the maximum score, with only $20 \%$ of patients comply with the recommended intake of these four components.

As shown in Table 3, patients in quartile 4 were less likely to be overweight as compared to patients in quartile 1 after adjusting for demographic and medical characteristics [Model $1: \mathrm{OR}=0.36 ; 95 \% \mathrm{Cl}=0.13,0.99$ ]. An additional variable of moderate-to-vigorous physical activity did not substantially change this significant association. In model 1, higher diet quality was associated with lower odds of low serum hemoglobin in quartile 3 . The significance value was reduced after adjusting for moderate-to-vigorous physical activity and overweight [Model 2: $\mathrm{OR}=0.35 ; 95 \% \mathrm{Cl}=0.13,0.92]$. In quartile 4 , the better diet quality was significantly showed with improved serum haemoglobin only after adjusting for behavioural factors [Model 2: $\mathrm{OR}=0.36 ; 95 \% \mathrm{Cl}=0.13,0.98]$.

\section{Discussion}

This study investigated the associations of diet quality with overweight and treatment-related side effects among breast cancer patients undergoing treatment. In this study, the consumption of whole grains, dairy, fatty acids and refined grains were low. This finding was consistent with earlier studies ${ }^{21,22}$ Avoidance and abstinence of certain foods are common among cancer patients, owing to belief that animal products is the cause of cancer development ${ }^{17,18}$ 
or treatment-related side effects that alter the taste perception ${ }^{23}$. On the other hand, dairy products are not habitual intakes of Malaysian ${ }^{29}$, which may further attribute to the low consumption pattern of dairy products among the patients.

Despite dairy products are good sources for protein, multivitamins and minerals which are essential for bodily function, breast cancer patients may limit their dairy intake attributed to the concern about the safety of growth hormone usage in animal products ${ }^{18}$. Lu et al. (2016) reviewed that the detrimental effect of dairy products on cancer mortality risk is dependent on type of dairy products. In this regards, only whole dairy food was found to link with higher mortality risk for prostate cancer ${ }^{30}$, while ensuring the safety consumption of skim or low fat dairy products, which may because of the cancer-promoting substance, luxuriant calcium ${ }^{31}$ or its high content of saturated fat ${ }^{32}$. Contrary to popular belief, the intake of whole grain products that commonly known as healthy food was reduced in breast cancer patients. During treatment, cancer patients experience various side effects which could interfere with the ability of consuming a balanced diet ${ }^{23,33,34}$, in spite of the high awareness of practising healthy eating. The compliance towards refined grain guideline could be deduced from the assessment of whole grains, where both of these components yield identical interpretation. As compared to whole grains, breast cancer patients prefer refined grains products with softer texture such as white rice, white bread or any food made with white flour. With regards to fatty acid component, the low score is originated from inadequate consumption of mono- and poly-unsaturated fatty acids. The individual component of saturated fat reflected that patients limited the food that are high in saturated fat, however, they did not adhere to the guidelines for unsaturated fatty acids. Although there is limited information regarding the fatty acid ratio, a consistent finding of the good compliance with saturated fatty acids intake was documented ${ }^{21,22}$. Polyunsaturated fatty acids from marine sources are suggested to ameliorate treatment-related side effects and improve treatment efficacy via antineoplastic effect ${ }^{35}$. In addition to saturated fatty acid, it is essential to assess the patients' intake of unsaturated fatty acids particularly during treatment. The biggest barrier to adherence to healthy fats recommendation could be high food prices ${ }^{36}$, for instance, margarines with low saturated fat are more expensive than butter that high in saturated fat. Furthermore, Malaysians hardly consume raw vegetables or fruits accompanied with oil-based dressings, which is not their ordinary food habit.

Current finding demonstrated that a better diet quality has protective effect against overweight among breast cancer patients, as supported by Custódio et al. $(2016)^{22}$. Similar relationship between diet quality and obesity has been widely reported by public health studies ${ }^{37-39}$. On the other hand, the finding of the relationship between diet quality and cancer risk or mortality was not consistent ${ }^{11,40}$. As cancer is a multi-site disease with different treatment, the relationship of diet with cancer prognosis should be studied according to various cancer types, whereby the development of cancer site-specific diet quality tools is deemed necessary. As previous studies indicated the negative impact of obesity on quality of life ${ }^{41}$ and survival ${ }^{4,5}$ of cancer patients, being overweight is not a desirable outcome for cancer patients who are usually considered to be malnourished. Adopting a healthy eating habit should be a bedrock of lifestyle strategy to achieve a normal weight range as well as to maintain an overall good health. Drenowatz et al. (2014) highlighted that high consumption of protein, sodium and empty calories were the contributors of overweight or obesity among healthy adults ${ }^{38}$, indicating the necessity of achieving an adequate, balanced, moderate and varied diet. In view of the significant role of healthy eating in disease prognosis, the utilization of diet quality indices is relatively pragmatic for breast cancer patients.

Iron status of cancer patients should be closely monitored as the incidence of anemia could compromise greatly their quality of life ${ }^{42}$. Current finding showed that higher quality diet was associated with reduced risk of low serum hemoglobin only after adjusting for physical activity and BMI (Model 2), suggests that diet quality might indirectly improve anemia by reducing excess body weight. It is noteworthy that the low serum hemoglobin could be owing to multiple nutrient deficiencies ${ }^{43,44}$ rather than a single nutrient. Vegetables and soy food that rich in iron or components enhancing iron absorption were found to associate positively with serum hemoglobin among middle-aged adults and elderly population ${ }^{44,45}$. Vitamin $\mathrm{C}$, in addition to iron, was found to be essential to prevent iron deficiency for obese patients who undergone bariatric surgery ${ }^{46}$. Vegetables and soy groups are mainly included in Healthy Eating Index assessment, elucidating the necessity of consuming these components in line with the dietary guidelines. Likewise, Mardas et al. (2017) showed that a whole food rather than a single nutrient was related with chemotherapy-induced gastrointestinal symptoms ${ }^{47}$. Iron rich food or dietary enhancers of iron absorption could be somehow beneficial to anemic cancer patients, despite the low hemoglobin level is generally attributable to cancer or treatment-related factors. With regards to the incidence of anemia, Thomson et al. (2011) recommended to keep body weight within a healthy range ${ }^{43}$. Inadequate diet intake could predispose patients to iron deficiency while obese women are likely to experience interrupted iron absorption ${ }^{43,45}$. In view of the potential inter-relationship between health indicators, investigation on the mediating effect of body mass index in respect to the association between diet quality and low serum hemoglobin are warranted.

Breast cancer patients were shown to be more likely to adhere to dietary guidelines within the shorter duration of diagnosis. Despite earlier studies demonstrated non-significant relationship of diagnosis time with diet quality in mixed cancer population or survivors ${ }^{13,48}$, such relationship was implicitly supported by the study of Custódio et al. (2016) ${ }^{22}$. Over the cancer treatment, a decline in diet quality was evident at the intermediate cycle of chemotherapy ${ }^{22}$. In general, poor diet quality is attributed to lack of food varieties or an imbalanced diet. As a result of cancer treatment, nutrition impact symptoms, for instance, taste changes and nausea ${ }^{23}$ could act as barriers to consuming a good quality diet. In making a decision for treatment plan, comorbidity is always a major issue for cancer patients in light of its potential dual burden on quality of life and survival ${ }^{49}$. In the present study, patients with comorbidities were shown to have poorer diet quality. Noting of the non-prospective study design, this finding does not preclude the notion of substantial impact of poor diet quality on the burden of multiple health outcomes.

Present study yields new insights into how a quality diet was linked to the clinical outcomes of patients with breast cancer, with the evaluation of the degree of conformance in consumption of various food groups with dietary guidelines. Achieving a healthy weight range has been set as a primary goal to prevent nutrient deficiencies and to ensure an optimal health for cancer population ${ }^{50}$. The assessment of diet quality in breast cancer patients, particularly those receiving treatment, therefore, provides valuable information for the identification of patients' nutritional status during this critical phase. Moreover, the association between diet quality and treatment-related side effects was performed using multivariate analysis, together with the adjustment of multiple confounders. This minimizes the bias that resulted from the factors that are out of study interest. 
The limitations of this study included cross sectional design, whereby, the causal effect of poor diet towards treatment-related side effects could not be assured. Despite Healthy Eating Index was found to be a well-established tool to predict obesity ${ }^{51}$, it does not consider the key principle of balance, as referring that each food groups should be taken in right amount or proportion. In regard to this limitation, an underestimation of over-eating might be existed. For instance, though protein is important for muscle building, over-consumption of proteins can contribute significant calorie which could lead to overweight. Lack of nutrition information, particularly for saturated, mono- and polyunsaturated fatty acids, may underscore the component of fatty acid in overall diet quality evaluation. However, additional food databases with higher availability of food items or nutrients were utilized in order to minimize such bias. As the patientreported outcomes rely on cancer types and treatment factors, these findings may not generalize to other cancer groups or trajectory phases.

\section{Conclusions}

In conclusion, this study demonstrated significant associations between diet quality with overweight and low serum hemoglobin. Breast cancer patients with better quality of diet were less likely to be overweight and have abnormally low level of hemoglobin. Findings from this study enable the health professionals to better understand the overall dietary intake of breast cancer patients during treatment, providing significant contribution to the implementation of effective strategies in improving patients' nutritional status and cancer care. Nonetheless, more investigations are deemed necessary to identify the exact mechanism of how diet may influence treatment-related side effects among breast cancer patients.

\section{List Of Abbreviations}

MREC - Medical Research and Ethics Committee

IPAQ-SF - International Physical Activity Questionnaire - short form

FFQ - food frequencies questionnaires

HEI - Healthy eating index

BMI - Body mass index

\section{Declarations}

\section{Ethics approval and consent to participate}

Ethics approval was provided by Medical Research and Ethics Committee (MREC), Ministry of Health Malaysia and Ethics Committee for Research Involving Human Subject Universiti Putra Malaysia. Patients' written informed consents were obtained prior to study enrolment.

\section{Consent for publication}

The authors give the consent for this manuscript titled Nutrition Indicators, Physical Functions and Health-related quality of life to be published in BMC Nutrition.

\section{Availability of data and materials}

The datasets used and/or analysed during the current study are available from the corresponding author on reasonable request.

\section{Competing interests}

The authors declare no conflict of interest regarding the publication of this articles.

\section{Funding}

This work was supported by the University Putra Malaysia (grant number GP-IPS 9507400).

\section{Authors' contributions}

All the authors planned and designed the study. The data were collected and computed by Krystal NLS. Chan YM and Zalilah MS were involved in data analysis and interpretation. Krystal NLS prepared the manuscript and this manuscript was editing or reviewed by Chan YM and Zalilah MS.

\section{Acknowledgements}

Special thanks are dedicated to all patients and staffs of National Cancer Institute at Putrajaya, for the enthusiasm in data collection. The authors thank Dr Subashini Elangkovan from the Clinical Research Centre; Norshariza Jamhuri, Betti Sharina Mohd Haniff, Siti Nuraini Mohd Samwil, and Ng Wai Han from the 
Department of Dietetics, for assistance along the data collection.

\section{References}

1. Hébuterne X, Lemarié E, Michallet M, De Montreuil CB, Schneider SM, Goldwasser F. Prevalence of malnutrition and current use of nutrition support in patients with cancer. J Parenter Enter Nutr. 2014;38(2):196-204. doi:10.1177/0148607113502674

2. Pressoir M, Desné S, Berchery D, et al. Prevalence, risk factors and clinical implications of malnutrition in french comprehensive cancer centres. Br $J$ Cancer. 2010;102(6):966-971. doi:10.1038/sj.bjc.6605578

3. van den Berg MMGA, Winkels RM, de Kruif JTCM, et al. Weight change during chemotherapy in breast cancer patients: a meta-analysis. BMC Cancer. 2017;17(1):259. doi:10.1186/s12885-017-3242-4

4. Jiralerspong S, Goodwin PJ. Obesity and breast cancer prognosis: Evidence, challenges, and opportunities. J Clin Oncol. 2016;34(35):4203-4216. doi:10.1200/JC0.2016.68.4480

5. Picon-ruiz M, Morata-tarifa C, Valle-goffın JJ. Obesity and Adverse Breast Cancer Risk and Outcome: Mechanistic Insights and Strategies for Intervention. $A$ Cancer Journals Clin. 2017;67(5). doi:10.3322/caac.21405.

6. Tachi T, Teramachi H, Tanaka K, et al. The impact of outpatient chemotherapy-related adverse events on the quality of life of breast cancer patients. PLoS One. 2015;10(4):1-15. doi:10.1371/journal.pone.0124169

7. Bower JE, Sciences B, Cancer JC. HHS Public Access. Science (80-). 2014;11(10):597-609. doi:10.1038/nrclinonc.2014.127.Cancer-related

8. Lyman GH, Michels SL, Reynolds MW, Barron R, Tomic KS, Yu J. Risk of mortality in patients with cancer who experience febrile neutropenia. Cancer. 2010;116(23):5555-5563. doi:10.1002/cncr.25332

9. Kane HL, Halpern MT, Squiers LB, Treiman KA, McCormack LA. Implementing and Evaluating Shared Decision Making in Oncology Practice. CA Cancer J Clin. 2014;64(December):377-388. doi:10.3322/caac.21245.

10. Wirt A, Collins CE. Diet quality - What is it and does it matter? Public Health Nutr. 2009;12(12):2473-2492. doi:10.1017/S136898000900531X

11. Du M, Liu SH, Mitchell C, Fung TT. Associations between diet quality scores and risk of postmenopausal estrogen receptor-negative breast cancer: A systematic review. J Nutr. 2018;148(1):100-108. doi:10.1093/jn/nxx015

12. George SM, Neuhouser ML, Mayne ST, et al. Postdiagnosis diet quality is inversely related to a biomarker of inflammation among breast cancer survivors. Cancer Epidemiol Biomarkers Prev. 2010;19(9):2220-2228. doi:10.1158/1055-9965.EPI-10-0464

13. George SM, Ballard-Barbash R, Shikany JM, et al. Better Postdiagnosis Diet Quality Is Associated with Reduced Risk of Death among Postmenopausal Women with Invasive Breast Cancer in the Women's Health Initiative. Cancer Epidemiol Biomarkers Prev. 2014;23(4):575-583. doi:10.1158/1055-9965.EPI-131162

14. Terranova CO, Protani MM, Reeves MM. Overall Dietary Intake and Prognosis after Breast Cancer: A Systematic Review. Nutr Cancer. $2018 ; 70(2): 153-163$. doi:10.1080/01635581.2018.1412478

15. Jacobs DR, Gross MD, Tapsell LC. Food synergy: An operational concept for understanding nutrition. Am J Clin Nutr. 2009;89(5):1543-1548. doi:10.3945/ajcn.2009.26736B

16. Wu G. Dietary protein intake and human health. Food Funct. 2016;7(3):1251-1265. doi:10.1039/c5fo01530h

17. Beeken RJ, Williams K, Wardle J, Croker H. "What about diet?" A qualitative study of cancer survivors' views on diet and cancer and their sources of information. Eur J Cancer Care (Engl). 2016;25(5):774-783. doi:10.1111/ecc.12529

18. Thomson AK, Heyworth JS, Girschik J, Slevin T, Saunders C, Fritschi L. Beliefs and perceptions about the causes of breast cancer: a case-control study. BMC Res Notes. 2014;7:1-8.

19. Shaharudin SH, Sulaiman S, Shahril MR, Emran NA, Akmal SN. Dietary Changes Among Breast Cancer Patients in Malaysia. Cancer Nurs. $2013 ; 36(2): 1$. doi:10.1097/NCC.0b013e31824062d1

20. Yong HY, Shariff ZM, Kandiah M, et al. Diet and physical activity in relation to weight change among breast cancer patients. Asian Pac J Cancer Prev. 2014;15(1):39-44. doi:10.7314/APJCP.2014.15.1.39

21. Ceccatto V, Faria Di Pietro P, Nogueira Previdelli A, et al. Brazilian healthy eating index revised (BHEI-R) of women before and during adjuvant treatment for breast cancer. Nutr Hosp. 2014;30(5):1101-1109. doi:10.3305/nh.2014.30.5.7439

22. Custódio IDD, Marinho EDC, Gontijo CA, Pereira TSS, Paiva CE, De Maia YCP. Impact of chemotherapy on diet and nutritional status of women with breast cancer: A prospective study. PLoS One. 2016;11(6):1-21. doi:10.1371/journal.pone.0157113 
23. Marinho E da C, Custódio IDD, Ferreira IB, Crispim CA, Paiva CE, Maia YC de P. Impact of chemotherapy on perceptions related to food intake in women with breast cancer: A prospective study. PLoS One. 2017;12(11):1-14. doi:10.1371/journal.pone.0187573

24. World Health Organization. Regional Office for the Western Pacific. The Asia-Pacific Perspective: Redefining Obesity and Its Treatment. Sydney: Health Communications Australia.; 2000. doi:0-9577082-1-1

25. Institute for Public Health. National Health and Morbidity Survey 2014: Malaysian Adult Nutrition Survey Volume I: Methodology and General Findings.; 2014.

26. National Cancer Institute; National Institutes of Health. Overview and Background of The Healthy Eating Index. https://epi.grants.cancer.gov/hei/. Accessed July 19, 2018.

27. Fokeena WB, Jamaluddin R, Khaza'ai H. Development and Assessment of the Reliability and Validity of a Diet Quality Index in a Sample of Malaysian University Students. J Food Nutr Res Vol 4, 2016, Pages 251-257. 2016;4(4):251-257. doi:10.12691/JFNR-4-4-9

28. IPAQ IPAQ. Guidelines for data processing and analysis of the International Physical Activity Questionnaire (IPAQ): short and long forms. Http://WwwlpaqKiSe/ScoringPdf. 2005;(November):1-15.

29. Shahril MR, Sulaiman S, Shaharudin SH, Akmal SN. Healthy eating index and breast cancer risk among Malaysian women. Eur J Cancer Prev. 2013;22(4):342-347. doi:10.1097/CEJ.0b013e32835b37f9

30. Lu W, Chen H, Niu Y, Wu H, Xia D, Wu Y. Dairy products intake and cancer mortality risk: A meta-analysis of 11 population-based cohort studies. Nutr J. 2016;15(1). doi:10.1186/s12937-016-0210-9

31. Giovannucci E. Dietary influences of 1,25(OH)2 vitamin D in relation to prostate cancer: a hypothesis. Cancer Causes Control. 1998;9(6):567-582. http://www.ncbi.nlm.nih.gov/pubmed/10189042. Accessed March 21, 2019.

32. Norton K, Olds T, Australian Sports Commission. Anthropometrica: A Textbook of Body Measurement for Sports and Health Courses. UNSW Press; 1996. https://books.google.com.my/books?

id=Bkk8FuB0P4IC\&dq=Gore+CJ:+Physiological+tests+for+elite+athletes/Australian+Sport+Commission+Champaign,+IL:+Human+Kinetics\%3B+2000\&source: Accessed May 14, 2018.

33. Boltong A, Aranda S, Keast R, et al. A prospective cohort study of the effects of adjuvant breast cancer chemotherapy on taste function, food liking, appetite and associated nutritional outcomes. PLoS One. 2014;9(7):1-9. doi:10.1371/journal.pone.0103512

34. de Vries YC, van den Berg MMGA, de Vries JHM, et al. Differences in dietary intake during chemotherapy in breast cancer patients compared to women without cancer. Support Care Cancer. 2017;25(8):2581-2591. doi:10.1007/s00520-017-3668-x

35. D'Eliseo D, Velotti F. Omega-3 Fatty Acids and Cancer Cell Cytotoxicity: Implications for Multi-Targeted Cancer Therapy. J Clin Med. 2016;5(2):15. doi: $10.3390 / \mathrm{jcm} 5020015$

36. Rao M, Afshin A, Singh G, Mozaffarian D. Do healthier foods and diet patterns cost more than less healthy options? A systematic review and metaanalysis. BMJ Open. 2013;3(12). doi:10.1136/bmjopen-2013-004277

37. Livingstone KM, McNaughton SA. Diet quality is associated with obesity and hypertension in Australian adults: a cross sectional study. BMC Public Health. 2016;16(1):1-10. doi:10.1186/s12889-016-3714-5

38. Drenowatz C, Shook RP, Hand GA, Hébert JR, Blair SN. The independent association between diet quality and body composition. Sci Rep. $2014 ; 4: 1-6$. doi:10.1038/srep04928

39. Drewnowski A, Aggarwal A, Tang W, et al. Obesity, diet quality, physical activity, and the built environment: the need for behavioral pathways. BMC Public Health. 2016;16(1):1-12. doi:10.1186/s12889-016-3798-y

40. Potter J, Brown L, Williams RL, Byles J, Collins CE. Diet quality and cancer outcomes in adults: A systematic review of epidemiological studies. Int $J$ Mol Sci. 2016;17(7):1-30. doi:10.3390/ijms17071052

41. Fang P, Tan KS, Troxel AB, Rengan R, Freedman G, Lin LL. High body mass index is associated with worse quality of life in breast cancer patients receiving radiotherapy. Breast Cancer Res Treat. 2013;141(1):125-133. doi:10.1007/s10549-013-2663-2

42. Kanuri G, Sawhney R, Varghese J, Britto M, Shet A. Iron deficiency anemia coexists with cancer related anemia and adversely impacts quality of life. PLoS One. 2016;11(9):1-11. doi:10.1371/journal.pone.0163817

43. Thomson CA, Stanaway J, Neuhouser ML, et al. Nutrient Intake and Anemia Risk in the WHI Observational Study. J Am Diet Assoc. 2011;111(4):532-541. doi:10.1016/j.jada.2011.01.017

44. Zhang Q, Qin G, Liu Z, et al. Dietary balance index-07 and the risk of anemia in middle aged and elderly people in southwest China: A cross sectional study. Nutrients. 2018;10(2):1-12. doi:10.3390/nu10020162 
45. Song P, Li L, Man Q, Wang C, Meng L, Zhang J. Case-control study of anaemia among middle-aged and elderly women in three rural areas of China. BMJ Open. 2014;4(8). doi:10.1136/bmjopen-2013-004751

46. Mischler RA, Armah SM, Wright BN, Mattar SG, Rosen AD, Gletsu-Miller N. Influence of diet and supplements on iron status after gastric bypass surgery. Surg Obes Relat Dis. 2016;12(3):651-658. doi:10.1016/j.soard.2015.09.007

47. Mardas M, Madry R, Stelmach-Mardas M. Link between diet and chemotherapy related gastrointestinal side effects. Wspolczesna Onkol. 2017;21(2):162167. doi:10.5114/wo2017.66896

48. Kane K, llic S, Paden H, et al. An evaluation of factors predicting diet quality among cancer patients. Nutrients. 2018;10(8):1-10. doi:10.3390/nu10081019

49. Sarfati D, Koczwara B, Jackson C. The impact of comorbidity on cancer and its treatments. CA Cancer J Clin. 2016;00(0):1-13. doi:10.3322/caac.21342.

50. Rock CL, Doyle C, Demark-Wahnefried W, et al. Nutrition and Physical Activity Guidelines for Cancer Survivors. CA Cancer J Clin. 2012;00:000-000.

doi:10.3322/caac. 21142

51. Asghari G, Mirmiran P, Yuzbashian E, Azizi F. A systematic review of diet quality indices in relation to obesity. Br J Nutr. 2017;117(8):1055-1065. doi:10.1017/S0007114517000915

\section{Tables}

Due to technical limitations, tables are only available as a download in the supplemental files section.

\section{Figures}

Figure 1: Radar graph of median components score in HEI-2015 and percentage of meeting dietary recommendations

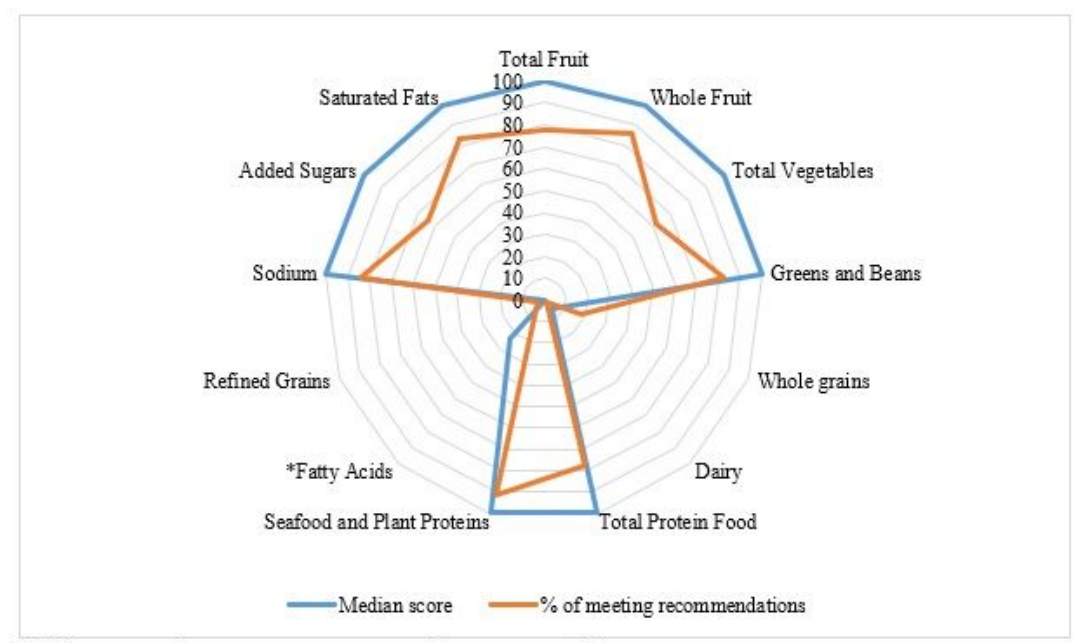

Median scores of component were expressed in percentage (\%)

Higher $\%$ indicate better conformance towards dietary guidelines

*Fatty Acids: ratio of poly- and mono-unsaturated fatty acids to saturated fatty acids

\section{Figure 1}

Radar graph of median components score in HEl-2015 and percentage of meeting dietary recommendations

\section{Supplementary Files}

This is a list of supplementary files associated with this preprint. Click to download.

- supplement1.pdf 